\title{
Microextração em sorvente empacotado (MEPS) para a determinação de fármacos em fluidos biológicos
}

\author{
Maria Eugênia Costa Queiroz \\ Faculdade de Filosofia Ciências e Letras de Ribeirão Preto, \\ Departamento de Química, Universidade de São Paulo, \\ Cep 14040-901, Ribeirão Preto, SP, Brasil \\ e-mail: mariaeqn@ffclrp.usp.br
}

\section{Resumo}

A microextração em sorvente empacotado (MPES) é uma nova técnica de preparo de amostra que foi desenvolvida em 2004. Na verdade, a MEPS é a miniaturização dos volumes dos dispositivos sortivos da extração em fase sólida (SPE) convencional, de mililitros para microlitros. O principal destaque da MEPS é que a ordem de magnitude do volume do solvente utilizado para a eluição dos analitos no processo de extração é adequada para injeção direta em sistemas de cromatografia líquida, cromatografia gasosa ou eletroforese capilar, sem nenhuma modificação do instrumento. Esta revisão apresenta os fundamentos teóricos da técnica MEPS e suas aplicações. O procedimento de MEPS com suas diferentes etapas (preparo da amostra, préconcentração dos analitos, limpeza do sorvente e eluição dos analitos) é discutido para amostras biológicas. Uma breve discussão geral das recentes aplicações da MEPS é também apresentada para determinação de fármacos e seus metabólitos em diferentes matrizes biológicas (plasma, urina, sangue, cabelo) para fins de monitorização terapêutica, controle de doping, análises de biomarcadores e toxicologia forense.

Palavras-chave

Microextração em sorvente empacotado; fármacos; amostras biológicas.

\section{Microextraction by packed sorbent (MEPS) for determination of drugs in biological fluids}

\section{Abstract}

Microextraction by packed sorbent (MEPS) is a new sample preparation technique developed in 2004 . Actually, MEPS is a miniaturization of conventional solid phase extraction (SPE) packed bed devices from mililitre bed volumes to microlitre volumes. The key factor in MEPS is that the volume of solvent used to elute the analytes from the extraction process is of a suitable order of magnitude to be injected directly on-line into Liquid Chromatography, Gas Chromatography or Capillary Electrophoresis systems, without any modification of the instrument. This review focuses on MEPS theory and applications. MEPS procedure with different steps (sample preparation, analytes pre-concentration, sorbent washing, and analytes elution) is discussed for biological samples. A short overview of recent and successful applications of MEPS for determination of drugs and their metabolites in different biological matrices (plasma, urine, blood, hair) for therapeutic drug monitoring, doping inspection, biomarkers analysis, and forensic toxicology are also summarized.

Keywords

Microextraction by packed sorbent; drugs; biological samples. 


\section{Introdução[1-12]}

A microextração em sorvente empacotado (MEPS) é uma recente técnica de preparo de amostra desenvolvida em 2004. Essa técnica consiste na miniaturização da técnica convencional de extração em fase sólida (SPE), na qual os volumes das amostras e dos solventes (eluentes) foram reduzidos de mililitros para microlitros (10-1000 $\mu \mathrm{L})$.

Na MEPS, uma microcoluna $(\approx 1 \mathrm{~cm} \times 0,2 \mathrm{~mm}$ d.i.), com aproximadamente 1-2 mg de material sólido empacotado, é conectada à agulha de uma microsseringa (100 - $250 \mu \mathrm{L})$, ou seja, integrada à microsseringa, como um plug com filtros polietileno em ambos os lados, Figura 1.

Diferentes fases seletivas adsorventes ou absorventes, ou seja, com diferentes mecanismos de extração, estão disponíveis no comércio, tais como: sílica (fase normal), dissilano (C2), octilsilano (C8), octadecilsilano (C18) - fases reversas, trocador de cátions forte (SCX), trocador de ânions forte (SAX) e fase mista M1 (C8 + SCX). No entanto, a diversidade de fases extratoras para MEPS disponíveis no comércio é muito inferior à variabilidade de fases para SPE. Os materiais sorventes para MEPS, geralmente, apresentam partículas irregulares de aproximadamente $50 \mu \mathrm{m}$ e porosidade $60 \AA$.

Em razão da miniaturização do sistema de extração, na MEPS, em uma única etapa, os solutos sorvidos na microcoluna são eluídos (dessorvidos) da fase extratora com alguns microlitros de solvente orgânico ou de fase móvel e diretamente injetados no sistema analítico. Esta etapa do processo de extração é o grande destaque da técnica de MEPS, ou seja, a ordem de magnitude do volume do solvente de dessorção $(\mu \mathrm{L})$ é adequada para introdução (injeção) direta deste eluente em sistemas cromatográficos. Desta forma, a técnica de MEPS tem sido hifenada às técnicas de separação, tais como: a cromatografia líquida de alta eficiência (HPLC), cromatografia gasosa (GC) ou a eletroforese capilar (CE).
Podemos também destacar a hifenação das técnicas de MEPS e espectrometria de massas. A técnica de MEPS foi utilizada para a pré-concentração de drogas de abuso (cocaína e seus metabólitos) de amostras de urina para análise direta em espectrômetro de massas com detector time-of-flight (DART-TOF). Dentre os vários sorventes avaliados (C8: octilsilano, ENV: poliestireno -divinilbenzeno hidroxilado, MCX: (divinilbeneno-N-polivinil-pirrolidona) polissulfônico, DAU: trocador iônico), a fase DAU apresentou os melhores resultados, ou seja, extrações mais eficientes que resultaram em espectros de massas com excelente razão sinal/ruído. A combinação das técnicas de MEPS/DART-TOF (Figura 2) resultou em análise rápida e grande confiabilidade na identificação dos analitos e seus isótopos ${ }^{[8]}$.
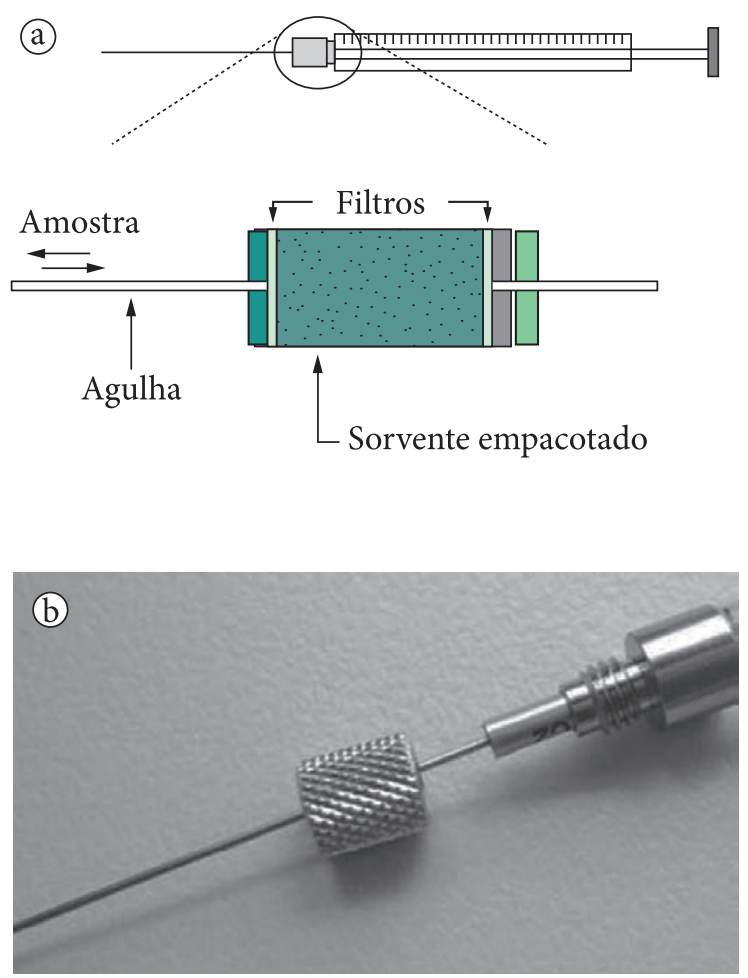

Figura 1 a) Esquema da microsseringa MEPS com microcoluna com material sorvente empacotado; e b) foto ilustrativa da microcoluna conectada à microsseringa [adaptada da referência n.1]. 


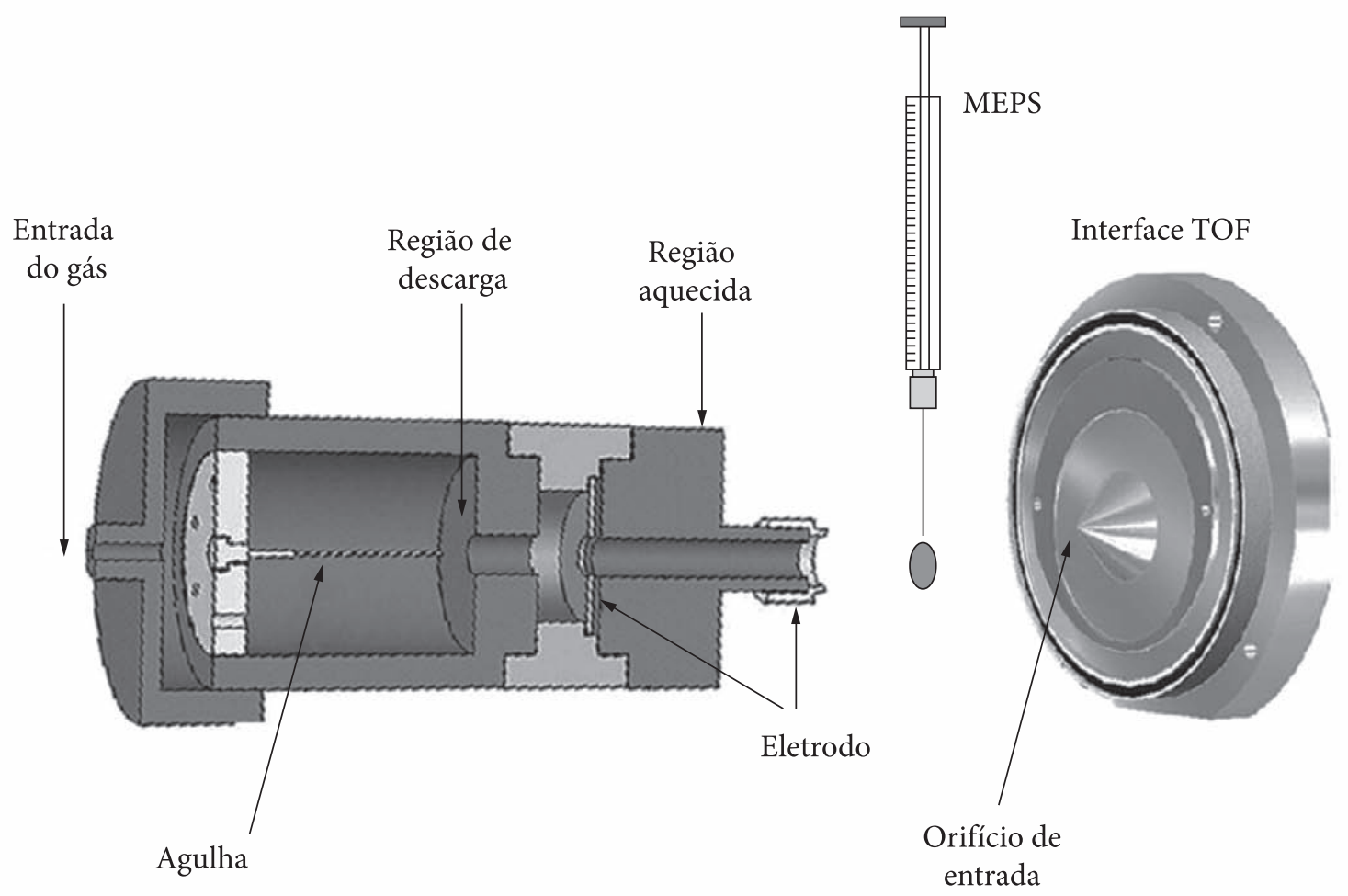

Figura 2 Esquema da combinação MEPS com análise direta em espectrômetro de massas DART-TOF [adaptada da referência n. 8].

O processo de MEPS (extração e introdução da amostra) pode ser realizado manualmente ou com auxílio do autoinjetor, procedimento em linha totalmente automatizado. As extrações (MEPS) automatizadas diminuem os coeficientes de variação interensaios (precisão analítica) e o tempo de extração para alguns minutos (1-2 minutos). O procedimento repetitivo de aspirar/dispensar a amostra por vários ciclos, para a pré-concentração dos analitos, quando realizado manualmente, tem sido descrito por alguns autores como uma desvantagem da técnica de MEPS.

Os cartuchos de SPE, geralmente são utilizados uma única vez e descartados, já as microcolunas de MEPS, dependendo da complexidade da amostra e dos cuidados do analista, podem ser reutilizadas de 50 a 100 vezes.

\section{Procedimento MEPS para a determinação de fármacos em fluidos biológicos ${ }^{[1-12]}$}

\subsection{Preparo das amostras biológicas}

Inicialmente, os fluidos biológicos $(10-250 \mu \mathrm{L})$ têm sido diluídos com solução tampão, para que os fármacos, geralmente ácidos fracos ou bases fracas, estejam na forma não ionizada (extração em fase reversa ou normal) ou ionizada (extração por troca iônica), favorecendo o processo de MEPS. As soluções tampão com valores de $\mathrm{pH}$ em intervalos extremos $(\mathrm{pH}<3$ ou $\mathrm{pH}>7)$ deverão ser evitadas, pois poderão danificar as fases sorventes à base de sílica. A precipitação das proteínas das amostras biológicas, com solventes orgânicos (acetonitrila ou metanol) ou com solu- 
ções ácidas aquosas ou soluções ácidas orgânicas, tem sido outro procedimento também realizado no pré-preparo das amostras biológicas.

Esses procedimentos descritos minimizam o efeito da matriz biológica no procedimento de MEPS, ou seja, minimizam a sorção (algumas vezes irreversível) dos compostos endógenos na fase extratora, resultam em cromatogramas mais limpos, com menores ruídos na linha de base e aumentam o número de vezes de reutilização dos sorventes de MEPS. A diluição da amostra biológica diminui a viscosidade da amostra, consequentemente favorece a transferência de massas dos solutos para a fase extratora.

\subsection{Pré-concentração dos fármacos}

Anterior ao procedimento de extração, a fase extratora tem sido condicionada ou ativada com alíquotas, respectivamente, de metanol e água (por exemplo, $3 \times 100 \mu \mathrm{L}$ ). Após esse procedimento, com o auxílio da microsseringa de MEPS, os fármacos são sorvidos na fase extratora da microcoluna, através dos processos de aspirar/dispensar alíquotas da amostra $(20-250 \mu \mathrm{L})$, no mesmo frasco que contém o fluido biológico, ou aspirar a amostra do frasco e dispensar no descarte.

A pré-concentração dos solutos tem sido realizada em um único ciclo aspirar/dispensar ou em vários. $\mathrm{O}$ aumento do número de ciclos resulta em aumento linear das taxas de recuperação dos solutos. Geralmente, as extrações de MEPS têm sido realizadas com 4 ciclos aspirar/ dispensar de $50 \mu \mathrm{L}$ de amostra. A seleção do sorvente é um fator importante para a obtenção de altas taxas de recuperação, por exemplo: as fases C2-C18 são adequadas para extração de solutos lipofílicos, já as fases poliméricas, tais como o poliestireno-divilnilbenzeno com grupos iônicos quimicamente ligados (troca iônica), ou as fases mistas (C8 + SCX) são adequadas para as determinações de solutos ácidos ou básicos.

\subsection{Eliminação dos interferentes}

Após a pré-concentração dos fármacos, a fase sólida é lavada com solvente $(50 \mu \mathrm{L})$, tais como água, solução ácida (exemplo, 0,1\% ácido fórmico), soluções tampão ou misturas de solução aquosas com solvente orgânico (metanol ou isopropanol), para remoção dos compostos endógenos. Nesta etapa de limpeza, a concentração de solvente orgânico, assim como o pH das soluções tampão deverão ser considerados para evitar ou minimizar a perda do soluto. $\mathrm{O}$ aumento da porcentagem de solvente orgânico na solução, geralmente, aumenta a perda do soluto.

\subsection{Elvição dos fármacos}

Os fármacos sorvidos na fase sólida são eluídos com alíquotas (20-50 $\mu \mathrm{L})$ de solvente orgânico ou de fase móvel e diretamente injetados no sistema analítico. $\mathrm{O}$ ajuste do $\mathrm{pH}$ da solução eluente (ionização dos solutos) é um fator importante para a obtenção de altas taxas de extração. $\mathrm{O}$ aumento do volume do eluente e da porcen-

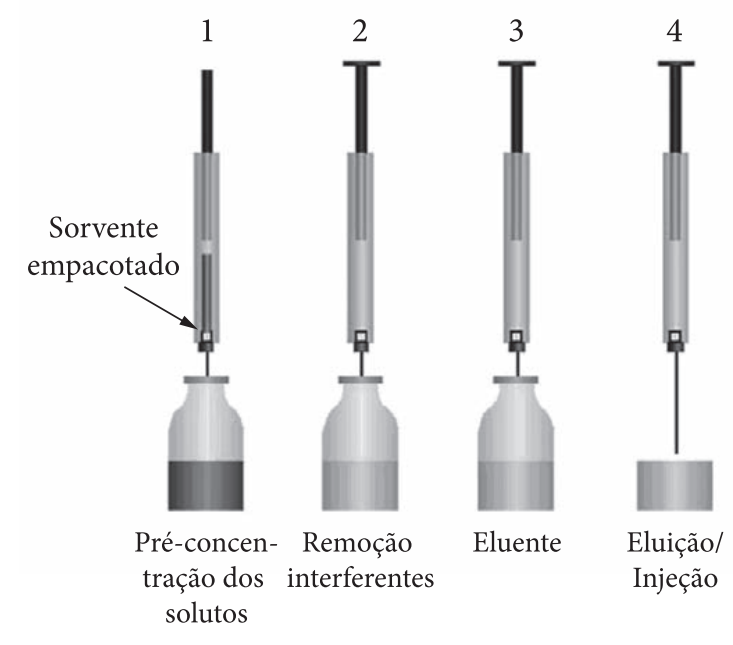

Figura 3 Processo MEPS com suas diferentes etapas, amostragem, pré-concentração dos solutos, eliminação dos interferentes, elvição dos solutos e introdução do extrato no sistema analítico. 
Tabela 1 Aplicações da MEPS associada a diferentes técnicas analíticas para a determinação de fármacos e seus metabólitos em fluidos biológicos.

\begin{tabular}{|c|c|c|c|c|}
\hline $\begin{array}{l}\text { Fármacos } \\
\text { (fluido biológico) }\end{array}$ & $\begin{array}{c}\text { MEPS } \\
\text { (Observações) }\end{array}$ & $\begin{array}{l}\text { Técnica } \\
\text { analítica }\end{array}$ & Linearidade & Referências \\
\hline $\begin{array}{l}\text { Ropivacaina e } \\
\text { metabólitos } \\
\text { (urina) }\end{array}$ & $\begin{array}{l}\text { FE: poliestireno } \\
\text { Urina: } 50 \mu \mathrm{L}(3 \mathrm{x}) \\
\text { Lavagem: } 50 \mu \mathrm{L} \text { água } \\
\text { Eluição: } 0,2 \% \mathrm{NH}_{4} \mathrm{OH} \text { em metanol }(20 \mu \mathrm{L})\end{array}$ & LC-MS/MS & 2-2000 nmol. . - & $\begin{array}{l}\text { M. Abdel-Rehim } \\
\text { et al. }(2004)^{[2]}\end{array}$ \\
\hline $\begin{array}{l}\text { Busulfan } \\
\text { (plasma) }\end{array}$ & $\begin{array}{l}\text { FE: poliestireno } \\
\text { Plasma: } 25 \mu \mathrm{L} \\
\text { (MEPS: } 1 \text { minuto versus LLE: } 40 \text { minutos) }\end{array}$ & LC-MS & $5-2500 \mathrm{ng} \cdot \mathrm{mL}^{-1}$ & $\begin{array}{l}\text { M. Abdel-Rehim } \\
\text { et al. }(2007)^{[3]}\end{array}$ \\
\hline $\begin{array}{l}\text { Acebutolol e } \\
\text { metoprolol } \\
\text { (plasma e urina) }\end{array}$ & $\begin{array}{l}\text { FE: poliestireno } \\
\text { Fluido biológico: } 50 \mu \mathrm{L}\end{array}$ & LC-MS/MS & $1-100 \mathrm{ng} \cdot \mathrm{mL}^{-1}$ & $\begin{array}{l}\text { M. Abdel-Rehim } \\
\text { et al. }(2007)^{[4]}\end{array}$ \\
\hline $\begin{array}{l}\text { Dopamina e } \\
\text { serotonina } \\
\text { (urina) }\end{array}$ & $\begin{array}{l}\text { FE: C8 (reutilizado } 300 \text { vezes) } \\
\text { Fluido biológico: } 50 \mu \mathrm{L} \\
\text { (Carry-over : } 0,1-0,2 \% \text { ) }\end{array}$ & LC-MS/MS & $50-4000 \mu \mathrm{g} \cdot \mathrm{mL}^{-1}$ & $\begin{array}{l}\text { M. Abdel-Rehim } \\
\text { et al. }(2007)^{[5]}\end{array}$ \\
\hline $\begin{array}{l}\text { Metanfetamina } \\
\text { e anfetamina } \\
\text { (cabelo) }\end{array}$ & $\begin{array}{l}\text { FE: } \\
\text { Cabelo: } 1 \mathrm{mg} \\
\text { Acetilação aquosa-MEPS } \\
\text { Eluição/Injeção (direta): } 50 \mu \text { Letanol - GC-MS (PTV) }\end{array}$ & GC-MS & $0,20-50 \mathrm{ng} \cdot \mathrm{mg}^{-1}$ & $\begin{array}{l}\text { Miyaguchi et al. } \\
\qquad(2009)^{[6]}\end{array}$ \\
\hline $\begin{array}{c}\text { Ciclofosfamida } \\
\text { (sangue total - rato) }\end{array}$ & $\begin{array}{l}\text { FE: poliestireno } \\
\text { Plasma: } 20 \mu \mathrm{L} \text { (diluição } 5 \mathrm{x}-0,1 \% \mathrm{HCOOH} \text { ) }\end{array}$ & LC-MS/MS & $0,5-150 \mu \mathrm{g} \cdot \mathrm{mL}^{-1}$ & $\begin{array}{l}\text { M. Abdel-Rehim } \\
\text { et al. }(2008)^{[7]}\end{array}$ \\
\hline $\begin{array}{l}\text { Cocaína e } \\
\text { metabólitos } \\
\text { (urina) }\end{array}$ & $\begin{array}{l}\text { FE: C8 (apolares-moderadamene polares), ENV } \\
\text { (alifáticos e aromáticos), MCX, DAU (básicos e ácidos) } \\
\text { Urina: } 100 \mu \mathrm{L}\end{array}$ & DART-TOF & $65-1200 \mathrm{ng} \cdot \mathrm{mL}^{-1}$ & $\begin{array}{l}\text { M. Abdel-Rehim } \\
\text { et al. }(2009)^{[8]}\end{array}$ \\
\hline $\begin{array}{l}\text { Fluoroquinolonas } \\
\text { (urina) }\end{array}$ & $\begin{array}{l}\text { FE: C18 } \\
\text { Urina: } 48 \mu \mathrm{L} \text { - diluída } 2,5 \% \text { ácido fórmico } \\
\text { Eluição: } 5 \% \text { ácido fórmico em metanol }\end{array}$ & CE-MS & $6,3-10,6 \mu \mathrm{g} \cdot \mathrm{L}^{-1}$ & $\begin{array}{l}\text { Valcárcel et al. } \\
\qquad(2009)^{[9]}\end{array}$ \\
\hline $\begin{array}{l}\text { Antidepressivos } \\
\quad \text { (plasma) }\end{array}$ & $\begin{array}{l}\text { FE: (C8 + SCE) } \\
\text { Plasma: } 250 \mu \mathrm{L}(3 \mathrm{x}) \\
\text { Lavagem: } 100 \mu \mathrm{L} \text { sol. ác fórmico 0,1\% } \\
\text { Elvição: fase móvel ( } 150 \mu \mathrm{L})\end{array}$ & HPLC-UV & $10-1000 \mathrm{ng} \cdot \mathrm{mL}^{-1}$ & $\begin{array}{l}\text { Queiroz et al. } \\
\qquad(2010)^{[10]}\end{array}$ \\
\hline $\begin{array}{l}\text { Atorvastatin e } \\
\text { metabólitos } \\
\text { (soro) }\end{array}$ & $\begin{array}{l}\text { FE: C8 } \\
\text { Soro: } 50 \mu \mathrm{L} \\
\text { Lavagem: Acetato amônio } \mathrm{pH} 4,5(100 \mu \mathrm{L}) \\
\text { Eluição: acetonitrila: Acetato amônio pH 4,5 (100 } \mu \mathrm{L}) \\
\text { PTFE filtro } 0,20 \mu \mathrm{m}\end{array}$ & $\begin{array}{l}\text { UHPLC-MS/ } \\
\qquad \text { MS }\end{array}$ & $0,5-100 \mathrm{nM}$ & $\begin{array}{l}\text { Nováková et al. } \\
(2011)^{[1]]}\end{array}$ \\
\hline $\begin{array}{l}\text { Clozapina e } \\
\text { metabólitos } \\
\text { (sangue - dried } \\
\text { spots) }\end{array}$ & $\begin{array}{l}\text { FE: C8 } \\
\text { Sangue: } 25 \mu \mathrm{L} \text { (diluição tampão fosfato) } \\
\text { Lavagem: água e sol. água + metanol } \\
\text { Eluição: fase móvel }\end{array}$ & $\begin{array}{l}\text { HPLC - } \\
\text { detector } \\
\text { coulométrico }\end{array}$ & 2,5-1000 ng. $\mathrm{mL}^{-1}$ & $\begin{array}{l}\text { Raggi et al. } \\
(2011)^{[12]}\end{array}$ \\
\hline
\end{tabular}

FE: Fase extratora, LLE: extração líquido-líquido, PTV: vaporizador com programa de temperatura, C8: octilsilano, ENV: poliestirenodivinilbenzeno hidroxilado, MCX: (divinilbeneno-N-polivinil-pirrolidona) polissulfônico, DAU (trocador iônico), DART-TOF: análise direta em tempo real em espectrômetro de massas time-of-flight, SCE: forte trocador de cátions. 
tagem de solvente orgânico favorece a dessorção dos fármacos. Para definir o volume da solução eluente, a eficácia do processo de dessorção dos solutos e o volume da alça de amostragem do HPLC são os fatores considerados.

\subsection{Limpeza da fase sólida}

Para reutilizar as microcolunas de MEPS, estas são lavadas (interensaios) 3-4 vezes com água e 4-5 vezes com o solvente utilizado na etapa de eluição. Este procedimento minimiza o efeito de memória para valores menores que $0,2 \%$.

A Figura 3 ilustra o processo de MEPS com suas diferentes etapas, pré-concentração dos fármacos, eliminação dos interferentes, eluição e introdução do extrato no sistema analítico.

As variáveis da técnica de MEPS, (volume de amostra, $\mathrm{pH}$ da amostra, número de ciclos aspirar/dispensar, composição e volume dos solventes de lavagem (remoção interferentes) e de eluição, têm sido otimizadas não somente para aumentar a sensibilidade analítica, mas também para minimizar o volume da amostra, o consumo de solventes orgânicos, o efeito de memória e o tempo de análise.

\section{Aplicações da MEPS}

A Tabela 1 ilustra as aplicações da MEPS associada a diferentes técnicas analíticas para a determinação de fármacos e seus metabólitos em fluidos biológicos para diferentes fins.

\section{Considerações finais}

A amostragem/pré-concentração dos fármacos em microcolunas de MEPS e a associação das etapas de eluição (dessorção) dos solutos e injeção do extrato nos sistemas analíticos resultam não somente na minimização do volume dos fluidos biológicos, geralmente $50 \mu \mathrm{L}$, e do consumo de solvente orgânicos $(\mu \mathrm{L})$, mas também em rápido procedimento de preparo de amostra, adequado para análises de rotina de amostras biológicas. Podemos também destacar a minimização da exposição dos analistas aos fluidos biológicos e redução ou eliminação de resíduos de solventes orgânicos gerados no processo de extração.

Segundo os trabalhos da literatura apresentados (Tabela 1), os métodos desenvolvidos com as associações da MEPS com as técnicas analíticas, HPLC, UHPLC, GC, CE e DART-TOPF apresentaram seletividade, alta sensibilidade analítica e linearidade adequada para a determinação de fármacos e seus metabólitos em diferentes amostras biológicas (sangue total, plasma, urina, cabelo) para diferentes fins, tais como, monitorização terapêutica, screening de drogas de abuso, toxicologia forense, análises de biomarcadores, dentre outros.

\section{Referências}

1 Abdel-Rehim M. Recent advances in microextraction by packed sorbent for bioanalysis. Journal of Chromatography A 2010; 1217(16):2569. PMid:19811788. http://dx.doi. org/10.1016/j.chroma.2009.09.053

2 Abdel-Rehim M, Altun Z, Blomberg L. Microextraction in packed syringe (MEPS) for liquid and gas chromatographic applications. Part II - Determination of ropivacaine and its metabolites in human plasma samples using MEPS with liquid chromatography/tandem mass spectrometry. Journal of Mass Spectrometry 2004; 39:1488-93. PMid:15578759. http://dx.doi.org/10.1002/jms.731

3 Abdel-Rehim M, Hassan Z, Skansem P, Hassan M. Simultaneous Determination of Busulphan in Plasma Samples by Liquid Chromatographyelectrospray Ionization Mass Spectrometry Utilizing Microextraction in Packed Syringe (MEPS) as On-line Sample Preparation Method. Journal of 
Liquid Chromatography \& Related Technologies 2007; 30:3029. PMCid:1176066. http://dx.doi. org/10.1080/10826070701632337

4 El-Beqqali A, Kussak A, Blomberg L, AbdelRehim M. Microextraction in Packed Syringe/ Liquid Chromatography/Electrospray Tandem Mass Spectrometry for Quantification of Acebutolol and Metoprolol in Human Plasma and Urine Samples. Journal of Liquid Chromatography \& Related Technologies 2007; 30:575. PMCid:1176066. http://dx.doi.org/10.1080/10826070601093895

5 El-Beqqali A, Kussak A, Abdel-Rehim M. Determination of dopamine and serotonin in human urine samples utilizing microextraction online with liquid chromatography/electrospray tandem mass spectrometry. Journal of Separation Science 2007; 30(3):421-24. PMid:17396602. http://dx.doi. org/10.1002/jssc. 200600369

6 Miyaguchi H, Iwata YT, Kanamori T, Tsujikama K, Inoue $H$. Rapid identification and quantification of methamphetamine and amphetamine in hair by gas chromatography/mass spectrometry coupled with micropulverized extraction, aqueous acetylation and microextraction by packed sorbent. Journal of Chromatography A 2009; 1216(18):4063-70. PMid:19286186. http://dx.doi.org/10.1016/j.chroma.2009.02.093

7 Said R, Hassan Z, Hassan M, Abdel-Rehim M. Rapid and Sensitive Method for Determination of Cyclophosphamide in Patients Plasma Samples Utilizing Microextraction by Packed Sorbent Online with Liquid Chromatography-Tandem Mass Spectrometry (MEPS-LC-MS/MS). Journal of Liquid Chromatography \& Related Technologies 2008; 31:683. PMCid:1176066. http://dx.doi. org/10.1080/10826070701853867
8 Jagerdeo E, Abdel-Rehim MZ. Screening of cocaine and its metabolites in human urine samples by direct analysis in real-time source coupled to time-of-flight mass spectrometry after online preconcentration utilizing microextraction by packed sorbent. Journal of the American Society for Mass Spectrometry 2009;20(5):891-9. http://dx.doi.org/10.1016/j.jasms.2009.01.010

9 Morales-Cid G, Cardenas S, Simonet BM, Valcarcel M. Fully Automatic Sample Treatment by Integration of Microextraction by Packed Sorbents into Commercial Capillary Electrophoresis-Mass Spectrometry Equipment: Application to the Determination of Fluoroquinolones in Urine. Analytical Chemistry 2009; 81(8):3188-93. http://dx.doi.org/10.1021/ac900234j

10 Chaves AR, Leandro FZ, Carris JA, Queiroz MEC. Microextraction in packed sorbent for analysis of antidepressants in human plasma by liquid chromatography and spectrophotometric detection. Journal of Chromatography B 2010; 878(23):2123-9. http://dx.doi.org/10.1016/j.jchromb.2010.06.023

11 Vičková $\mathrm{H}$, Solichová $\mathrm{D}$, Bláha $\mathrm{M}$, Solich $\mathrm{P}$, Nováková L. Microextraction by packed sorbent as sample preparation step for atorvastatin and its metabolites in biological samples-Critical evaluation. Journal of Pharmaceutical and Biomedical Analysis 2011; 55(2):301-8. http:// dx.doi.org/10.1016/j.jpba.2011.01.025

12 Saracino MA, Lazzara G, Prugnoli B, Raggi MA. Rapid assays of clozapine and its metabolites in dried blood spots by liquid chromatography and microextraction by packed sorbent procedure. Journal of Chromatography A 2011; 1218(16):2153-9. http:// dx.doi.org/10.1016/j.chroma.2011.01.071

Recebido: 22/07/2011 Aceito: 02/08/2011 